\title{
EL SUJETO DE LA ENUNCIACIÓN: DIÁLOGOS ENTRE LA LINGÜÍSTICA Y EL PSICOANÁLISIS
}

\author{
Karina Savio* \\ Consejo Nacional de Investigaciones Científicas y Técnicas (CONICET) \\ Universidad de Buenos Aires \\ Facultad de Filosofía y Letras \\ Instituto de Lingüística \\ Buenos Aires, Argentina
}

\begin{abstract}
Resumen: Este artículo se propone realizar un recorrido de la expresión sujeto de la enunciación en la obra de Jacques Lacan. Dado que en los estudios en los que se aborda la problemática de la enunciación no existe un consenso en torno a qué sujeto se hace referencia, recuperamos el origen psicoanalítico de este término con el objetivo de aventurar algunas reflexiones. Este camino nos ha conducido, como primer paso, a rastrear la noción de sujeto en Benveniste, y luego, de enunciación en los textos de Lacan. El análisis ha determinado la coexistencia de tres vertientes en torno a la enunciación, que permiten introducir diferentes dimensiones respecto a cómo se concibe el sujeto de la enunciación.
\end{abstract}

Palabras claves: Psicoanálisis. Lacan. Sujeto. Enunciación.

\section{INTRODUCCIÓN}

La expresión sujeto de la enunciación es frecuentemente empleada en diversos estudios relativos al discurso y a la enunciación y, por ende, aparece en numerosos trabajos que se interrogan respecto a la construcción de este sujeto en el relevamiento de diferentes materiales. Sin embargo, no todos concuerdan con el modo en que esta es definida. Entre otros sentidos que se le atribuyen, en algunos casos, es utilizada como un sinónimo del término enunciador; en otros, reenvía al autor del texto. Ahora bien, ¿a qué nos referimos cuando recurrimos a esta expresión?, ¿qué implícitos conlleva la noción de sujeto?, ¿es el sujeto que pertenece a la enunciación, que está 'sujeto' a ella?, ¿es anterior a la enunciación?, ¿qué se desprende de su análisis? Es difícil, quizás, encontrar respuestas a estas preguntas que satisfagan las distintas orientaciones teóricas que hacen uso de ella, partiendo de la complejidad a la que hacemos alusión. Nos conformamos en este artículo con intentar bordear esta problemática articulando dos campos del saber que pueden pensarse en interlocución, aunque sin forzar su homologación: la lingüística ${ }^{1}$ y el psicoanálisis. Nos proponemos, en particular, indagar el modo en que el sujeto de la enunciación es presentado en la obra lacaniana, con el fin último de extraer de allí algunas conclusiones que nos permitan aventurar algunas reflexiones al respecto.

\footnotetext{
* Doctora en Letras. Psicóloga. Investigadora asistente del CONICET. Docente de la Universidad de Buenos Aires y de la Universidad Nacional Arturo Jauretche. E-mail: karinasavio@ fibertel.com.ar

${ }^{1}$ Concebimos aquí a la lingüística en un sentido amplio.
} 
El camino que aquí emprendemos no es azaroso. Normand $(1986,1997)$ señala la ausencia del sintagma sujeto de la enunciación en los trabajos de Benveniste. El sintagma, en verdad, fue empleado en primer lugar por filósofos y psicoanalistas. Incluso, a fines de los sesenta tenía una recepción mayor entre estos dos grupos que entre los lingüistas (Normand, 1985, 1997). Por otra parte, como señala muy tempranamente Kristeva (1971) -en un artículo en el que se pregunta por el sujeto de la lingüística- el vínculo entre el sujeto y el significante implica un conjunto de problemas comunes entre la lingüística y el psicoanálisis; problemas que hoy no han perdido valor.

Antes de comenzar a transitar este sendero que hemos planteado, es importante aclarar que no pensamos aquí en una teoría del sujeto, ni en las diferentes concepciones elaboradas en torno al sujeto y su relación con el lenguaje. En esta línea, Authier-Revuz (1984), recuperando el dialogismo bajtiniano, la noción de interdiscurso y la ruptura del psicoanálisis con la creencia de un sujeto dueño de su decir, refiere a la heterogeneidad constitutiva, relativa a la condición de existencia de ese sujeto, y a la heterogeneidad mostrada, asociada a las formas lingüísticas con las que el sujeto hablante negocia con esa otra heterogeneidad. Ambos planos son, según ella, diferentes, pero operan de manera solidaria. En este texto, centramos nuestro enfoque no en el sujeto en tanto condición de existencia de un discurso, sino en el sujeto que se desprende de la enunciación.

La relación entre el psicoanálisis y la lingüística es de larga data. Es conocida la cercanía de Lacan hacia la linguiística y hacia la filosofía del lenguaje, fundamentalmente hacia los trabajos de Saussure, Benveniste, Jakobson y Austin, entre otros; cercanía que luego será profundizada por autores como Milner (1980, 2003) y Arrivé (2001). En Argentina, a diferencia de otros países ${ }^{2}$, esta línea de investigación es aún incipiente, sobre todo desde los estudios lingüísticos.

En este escrito, para lograr nuestro objetivo, en primer lugar, hacemos referencia a la teoría enunciativa de Benveniste, puntualmente a la noción de sujeto que él menciona y desarrolla en algunos de sus textos. En segundo lugar, revisamos el término enunciación ya sea en los seminarios como en los escritos de Lacan para intentar clarificar el modo en que él entiende este concepto lingüístico. Sobre este punto nos apoyamos, luego, para presentar al sujeto de la enunciación desde la óptica lacaniana. Al igual que con la enunciación, rastreamos la emergencia de este sintagma en toda su obra, para, finalmente, interrogarnos sobre su uso desde la lingüística.

\section{EL ¿SUJETO? DE BENVENISTE}

Como ya hemos advertido en nuestra introducción, Benveniste no recurre a la expresión sujeto de la enunciación en sus trabajos, pero sí emplea el término sujeto en algunos de sus textos. Por este motivo, Normand se pregunta si el lingüista estaba en verdad preocupado en elaborar una teoría del sujeto (1986, p. 202). Uno podría responder rápidamente que, efectivamente, ese no era su principal interés. Todorov (2014, p. 219) afirma, en este sentido, que la concepción "austera" del ideal de la ciencia en Benveniste

\footnotetext{
${ }^{2}$ En Brasil, por ejemplo, podemos destacar el trabajo de Flores (1999).
} 
lo priva de considerar "elementos externos" al lenguaje: históricos, políticos, literarios, filosóficos. Psicoanalíticos, podríamos añadir nosotros. No obstante, su inclinación por considerar la subjetividad en el lenguaje lo conduce a remitirse -aunque en escasas oportunidades- a esta noción.

Según Normand $(1986,1997)$, el término sujeto designa en la obra de este autor el sujeto gramatical, el yo psicológico o ego filosófico, revisado por la fenomenología, y con frecuencia la figura de una persona, pero nunca involucra una entidad que podría corresponderse con el sujeto escindido del psicoanálisis. Sin embargo, para Kristeva (2014) la problemática de la subjetividad y de la intersubjetividad en el lenguaje que plantea Benveniste implica un sujeto más complejo que el sujeto cartesiano y "[p]or añadidura, y sin que quede de manifiesto, esboza una apertura en dirección al sujeto de lo "inconsciente"' (KRISTEVA, 2014, p. 25). En este artículo, intentaremos dar cuenta de que la lectura de los textos de este autor nos acerca más a esta última posición.

Cuatro son las nociones medulares con las que el sujeto benvenisteano puede ser articulado: el lenguaje, el discurso, el acto y el otro.

En primer lugar, el lingüista manifiesta que es en y por el lenguaje que el hombre se constituye como sujeto (BENVENISTE, 2004a, p. 180). Por tal razón, podríamos afirmar que para este autor no hay un sujeto previo al lenguaje, ya que este es condición necesaria para que emerja.

En segundo lugar, y como consecuencia de lo anterior, en la medida en que el sujeto requiere del lenguaje se puede sostener que es efecto de este lenguaje y que es allí donde se lo puede hallar. Pero esta localización no es en el lenguaje en tanto facultad del hombre, sino en su puesta en funcionamiento a través del discurso. Por ello, Benveniste plantea que "es en la instancia de discurso en que yo designa el locutor donde éste se enuncia como "sujeto"" 3(2004a, p. 182). En esta cita vemos que se establece una clara distinción entre el locutor y el sujeto. El locutor está puesto en relación con la apropiación (2004a, p. 184; 2004b, p. 84-85) y, podríamos pensar, entonces, con la intencionalidad, mientras que el sujeto, con el efecto. Por otra parte, en "Observaciones sobre la función del lenguaje en el descubrimiento freudiano", texto de 1968, se perfila tímidamente un sujeto dividido por este discurso: "hay antinomia en el sujeto entre el discurso y la lengua" (2004a, p. 78), asegura. Mientras que la lengua es pensada como sistema, universal para todos, el discurso es propio de cada uno.

En tercer lugar, en Benveniste el discurso está vinculado con la enunciación y, por ende, con el acto. Por un lado, la enunciación es concebida como aquella que supone la conversión individual de la lengua en discurso (2004b, p. 83-84). Por el otro, es definida como un acto: "es este poner a funcionar la lengua por un acto individual de utilización" (2004b, p. 83). Esto nos permite pensar que el sujeto no solo requiere del lenguaje, no solamente se localiza en el discurso, sino que también es fugaz: subsiste en el instante de ese acto, para luego desaparecer. El lingüista manifiesta: "Con que uno de los hombres los pronuncie, los asume, y el pronombre yo, de elemento de un paradigma, se trasmuta en una designación única y produce, cada vez, una persona nueva"4 (2004b, p. 71). A

\footnotetext{
${ }^{3}$ A menos que se aclare, los destacados de las citas son nuestros. En este caso, corresponde al original.

${ }^{4}$ El destacado es del autor.
} 
pesar de que en esta cita el término sujeto no es empleado, podría pensarse que esa "persona" a la que aquí se alude implica la aparición de un sujeto nuevo que nace en cada acto enunciativo.

Finalmente, el sujeto no puede comprenderse si no es en relación con el otro. En efecto, en el momento en el que el locutor se constituye como sujeto en su discurso, también instituye al otro. Así lo afirma Benveniste: "El lenguaje no es posible sino porque cada locutor se pone como sujeto y remite a sí mismo como yo en su discurso. En virtud de ello, yo plantea otra persona, la que, exterior y todo a "mí", se vuelve mi eco al que digo tú y que me dice tú" (2004b, p.181). Por tal sentido, refiere a una "realidad dialéctica" (2004b, p. 181) en el que ambos términos yo-tú se definen en su vinculación con el otro. De esta manera, considera que toda enunciación es, explícita o implícita, una alocución, postula un alocutario (2004b, p.84-85).

\section{LAS TRES ENUNCIACIONES LACANIANAS}

A los fines de reflexionar en torno a la propuesta lacaniana alrededor de la expresión sujeto de la enunciación, es necesario, como primer paso, esclarecer el modo en que el psicoanalista francés entiende la noción que subyace a ese sintagma, a saber, la enunciación. Por ello, hemos emprendido un recorrido por los diferentes lugares en los que Lacan emplea este término a lo largo de su enseñanza. Recordemos que en el apartado anterior habíamos determinado que Benveniste (2004b) define la enunciación como un acto, un acto de apropiación del enunciado, que inscribe al que habla en su habla. Esta definición articula, entonces, entre otras variables, el enunciado con la situación en la que se lo realiza.

El término enunciación aparece en la obra lacaniana en dos sentidos: por un lado, en un sentido amplio y general, remite a la acción de enunciar, y, por el otro, en un sentido restringido, recupera la referencia lingüística. Este último matiz, es decir, el matiz lingüístico, se registra por primera vez en 1956 durante el Seminario 3 (1990a). En seminarios posteriores, fundamentalmente, en Seminario 6 (2014), Seminario 7 (1990b), Seminario 9 (s.f.a) ${ }^{6}$, Seminario 11 (2001), Seminario 13 (s.f.c), Seminario 14 (s.f.d), Seminario 16 (2008f), Seminario 17 (2006), es una noción que retoma y desarrolla, para, luego, ir desapareciendo de su enseñanza gradualmente. En este sentido, su uso decrece a lo largo de los años, y el último seminario en el que es mencionado fugazmente es el Seminario 24 (s.f.f). En relación con los escritos, es abordado, principalmente, en textos publicados entre 1960 y 1963: “Observaciones sobre el informe de Daniel Lagache: 'Psicoanálisis y estructura de personalidad"” (2008d), "Kant con Sade” (2008b), "Subversión del sujeto y dialéctica del deseo en el inconsciente freudiano" (2008g) y "Posición del inconsciente" (2008e).

El término enunciación es utilizado por primera vez el 13 de junio de 1956 durante el Seminario 3 (1990a) dedicado a la psicosis. En la clase 22, en la que marca la diferencia

\footnotetext{
${ }^{5}$ Los destacados son del autor.

${ }^{6}$ Para los seminarios inéditos, usamos la traducción de la Escuela Freudiana de Buenos Aires. Ver Referencias bibliográficas.
} 
entre el otro en tanto semejante y el Otro en donde el yo (je) se constituye, plantea: "El yo (je) es el yo (je) del que pronuncia el discurso. Todo lo que se dice tiene bajo sí un yo (je) que lo pronuncia. En el interior de esa enunciación aparece el tû"7 (1990a, pp.391392). Señala, a su vez, que el yo y el tú no presentan una relación simétrica, recíproca. Esta articulación entre la enunciación y el plano de la intersubjetividad, en la que nos resuenan los planteos de Benveniste, será abandonada en los años siguientes.

Es el Seminario 6 (2014) dictado entre 1958 y 1959, que trata sobre el deseo y su interpretación, el que recoge la distinción entre enunciado y enunciación establecida desde la lingüística adecuándola a sus propios intereses teóricos. En efecto, en este seminario mientras que el enunciado queda ubicado en relación con lo dicho, la enunciación se presenta situada en referencia con el inconsciente. Esta articulación entre enunciación e inconsciente se mantendrá a lo largo de toda su obra. Veamos lo que indica en la primera oportunidad en la que menciona este par de términos:

\begin{abstract}
A partir del momento en que la estructura de la cadena significante ha realizado el llamado al Otro, es decir, donde el proceso de la enunciación se distingue de la fórmula del enunciado y se le superpone, la captura del sujeto dentro de la articulación de la palabra -captura que al principio era inocente- deviene inconsciente. (2014, p.25)
\end{abstract}

Como puede observarse en esta cita, aquí se distingue enunciado de enunciación. En esta clase el psicoanalista está reflexionando sobre la constitución del sujeto y ubica la enunciación en función de la captura del sujeto por el Otro, en la que pierde su inocencia.

Esta constitución está, por otra parte, asociada con el lenguaje, por lo que advierte una relación de dependencia entre el lenguaje y el hombre: "el hombre habla sin duda pero para hablar ha de entrar en el lenguaje y en su discurso preexistente" (2004, p.24). Tesis que nos conduce nuevamente al trabajo del lingüista francés.

En "Posición del inconsciente" (2008e), se retoma también esta asociación entre enunciación e inconsciente: "No podemos por consiguiente dejar de incluir nuestro discurso sobre el inconsciente en la tesis misma que enuncia, que la presencia del inconsciente, por situarse en el lugar del Otro, ha de buscarse en todo discurso, en su enunciación." (2008e, p.793).

Esta referencia al inconsciente, por un lado, permite entender las razones por las cuales la enunciación carga con un sentido "inicialmente oscuro" (2008f, p.92) y, por el otro, nos reenvía indefectiblemente a las nociones de Otro y de discurso. En efecto, en la enseñanza de Lacan, esta instancia es concebida, entre otras definiciones, como el discurso del Otro (LACAN, 2008a). Por tal razón, no sorprende que se establezca una analogía entre ese discurso y la enunciación:

\footnotetext{
El pensamiento es, ante todo, algo que participa de esa dimensión de lo no dicho que acabo de introducir por medio de la distinción entre el proceso de la enunciación y el proceso del enunciado. Pero, por supuesto, para que un no dicho sea un no dicho, hay que decir. Para que ese no dicho subsista, hay que decirlo en el nivel del proceso de la enunciación, es decir, en calidad de discurso del Otro (2014, p. 89)
}

\footnotetext{
${ }^{7}$ Los destacados pertenecen al original.
} 
Si bien el vínculo entre la enunciación y el inconsciente persiste inalterado en sus textos, encontramos que el modo en que alude a esta noción lingüística fluctúa en el transcurso de su enseñanza en torno a tres sentidos: por un lado, se la define como acto, proceso; por el otro, se la designa como nivel, dimensión, plano; por último, se la nombra como línea, cadena. En algunas oportunidades estas denominaciones se entrecruzan; por ejemplo, en el Seminario 6 se menciona el "nivel del proceso de la enunciación" (2014, p. 89). Antes de examinar estos tres grupos para analizar sus diferentes implicancias, adelantemos que nosotros leemos allí, en este cambio de nominación, distintas miradas sobre este fenómeno, que inciden sobre el modo de pensar el sujeto.

En primer lugar, siguiendo la definición que provee la lingüística, Lacan refiere a la enunciación en tanto acto en tres oportunidades del Seminario 6 (2014), en dos momentos del Seminario 9 (s.f.a) y en uno del Seminario 14 (s.f.d), y en tanto proceso siete veces en el Seminario 6 (2014). Mientras que acto es un término que aparece en Benveniste, recordemos que en la traducción francesa "Les embrayeurs, les cátegories verbales et le verbe russe" de Roman Jakobson (1963, p.181) -interlocutor del psicoanalista francés- se alude específicamente al "proceso de la enunciación”. Este primer sentido está arraigado, entonces, en el decir lingüístico, encuentra su origen allí, y puede vincularse con el plano del hacer y con el acontecimiento mismo de proferir un enunciado. Por otra parte, en tanto que el acto está relacionado puntualmente con la enunciación, el proceso se liga tanto con la enunciación como con el enunciado: de hecho, Lacan indica la existencia de un proceso que involucra la enunciación y un proceso que atañe el enunciado.

En segundo lugar, esta noción es denominada plano, nivel, dimensión. El primer caso se menciona una única vez en el Seminario 11 (2001). El término nivel es el que más insiste en las clases: seis veces en el Seminario 6 (2014); tres veces en el Seminario 9 (s.f.a); dos, en el Seminario 11 (2001); y dos, en el Seminario 16 (2008f). Dimensión es empleada en el Seminario 6 (2014) y en el Seminario 14 (s.f.d). Con respecto a esta segunda acepción, al igual que con la idea de proceso, Lacan postula dos planos/niveles/dimensiones: el del enunciado y el de la enunciación. Podría pensarse que estos dos planos se corresponderían con aspectos del lenguaje: aquel vinculado con el mensaje y aquel relativo a la dimensión inconsciente. Estos niveles del lenguaje están en sintonía, por cierto, con otra de las definiciones que Lacan realiza sobre el inconsciente, a saber: el inconsciente está estructurado como un lenguaje (2008a).

Por último, aunque con una menor frecuencia, la enunciación es entendida como una línea o una cadena. Mientras que el término línea se usa en el Seminario 6 (2014), la idea de cadena se rastrea en dos momentos del Seminario 16 (2008f) y en uno en "Observaciones sobre el informe de Daniel Lagache: 'Psicoanálisis y estructura de la personalidad" " $(2008 \mathrm{~d})^{8}$. En este sentido, la enunciación no es solamente un acto o una dimensión del lenguaje, como se presenta desde la lingüística, sino también un Otro enunciado. Si Lacan caracteriza el inconsciente -en sintonía con las definiciones anteriores-como una cadena de significantes (2008g, p.760), no sorprende, entonces, que

\footnotetext{
${ }^{8}$ Tanto la vertiente de la enunciación concebida como dimensión/plano/nivel, como aquella relativa a la cadena o línea podrían localizarse en el grafo del deseo (LACAN, 2008g, p.777). No lo desarrollamos aquí debido a la complejidad teórica del grafo y, por ende, a la extensión que nos llevaría su despliegue.
} 
la enunciación pueda ser vista como tal. Es aquí, podríamos pensar, el lugar donde radica la novedad de la propuesta psicoanalítica, en esta "duplicidad interna" (2014, p. 93). Veamos un ejemplo para clarificar esta vertiente:

\footnotetext{
Por lo que atañe al sueño, es importante que ustedes recuerden de qué modo nos lo comunican. Siempre lo hacen por medio de un enunciado. ¿Qué es lo que nos detalla el sujeto? Otro enunciado, pero no basta en absoluto con decir eso. Nos presenta ese otro enunciado como una enunciación. (2014, p. 106).
}

En este fragmento se señala que en el relato del sueño se origina otro enunciado que se manifiesta como enunciación, y que puede ponerse en relación con el sentido de esa formación del inconsciente.

Siguiendo esta misma línea, en el Seminario 17 (2006) se describe el enigma y la interpretación como una enunciación sin enunciado. Podría pensarse, en estos casos, que ambos se soportan en un enunciado, pero que este apunta a su enunciación.

Ahora bien, ¿cómo articula Lacan este par de términos, enunciado y enunciación? Para el psicoanalista, existe una relación de "discordancia" (1990b, p.81) entre el enunciado y la enunciación, lo que podría atribuirse a que sus estructuraciones son opuestas y discontinuas (2014). Incluso, en el Seminario 14 (s.f.d, p.36) plantea la existencia de un "intervalo" entre ambos. Sin embargo, a pesar de esta discrepancia, estas líneas se pueden cruzar en algún punto de la cadena. En el Seminario 9 (s.f.a), al analizar la paradoja de la expresión "yo miento", en la que si el sujeto miente, entonces, dice la verdad, aclara con respecto a esta cuestión que estas líneas se embrollan y se confunden. Es decir, en determinado momento la cadena de la enunciación tropieza con la cadena del enunciado, que refleja, en ese mismo movimiento, la enunciación que subyace.

\section{EL SUJETO DE LA ENUNCIACIÓN EN LACAN}

Antes de comenzar con el análisis de la expresión sujeto de la enunciación en la enseñanza de Lacan, debemos hacer un breve comentario sobre la noción de sujeto. Este término, casi inexistente en la obra de Freud, se erige como uno de los pilares que sostienen el edificio teórico del psicoanalista francés, apareciendo muy tempranamente en sus trabajos ${ }^{9}$. Esta trascendencia teórica y la magnitud de referencias sobre el sujeto en la obra lacaniana nos impide desarrollarlo sintética y cabalmente. Por este motivo, solamente precisaremos algunas cuestiones que nos serán de utilidad para reflexionar, luego, sobre el sujeto de la enunciación.

En primer lugar, es necesario retomar la diferencia entre la noción de sujeto y el yo que el psicoanalista establece en sus primeros trabajos. Mientras que el yo forma parte del registro de lo imaginario, el sujeto pertenece a la dimensión simbólica. Por esta razón, el yo posee cierta ilusión de unidad y el sujeto, en cambio, es un sujeto dividido, escindido por el significante; es, en fin, el sujeto del inconsciente.

\footnotetext{
${ }^{9}$ Para una ampliación sobre esta formación temprana, véase Ogilvie (2000).
} 
En segundo lugar, es interesante observar que el momento en que Lacan da inicio, según Le Gaufey (2010), a desarrollar una definición de sujeto que persistirá sin modificaciones en toda su enseñanza es el año en el que introduce la distinción entre enunciado y enunciación, es decir, 1959. En este año empieza a trabajar en una nueva mirada sobre el sujeto que culminará en 1961 con la conocida fórmula que introduce en el Seminario 9 (s.d.a): el significante representa al sujeto para otro significante. Esta definición, que es pasible de diversas lecturas, presenta algunos puntos a destacar. Por un lado, el sujeto no ocupa el lugar de agente, sino que es representado por un significante. Por el otro, para que sea representado, un significante debe faltar de la cadena, debe estar perdido. Por otra parte, el significante no lo representa para otra persona, sino para otro significante, lo que aleja al sujeto del plano de la intersubjetividad y lo articula con el lenguaje. En efecto, según el psicoanalista, el sujeto es un efecto del lenguaje.

Por último, queremos referirnos a la disyunción que Lacan establece entre el sujeto y el saber. En relación con esta caracterización, es el Seminario 9 (s.f.a) el primer seminario en el que el psicoanalista ubica el saber en el lugar del Otro, lo que deja al sujeto no sabiendo nada y, por ende, desprovisto de toda reflexividad (LE GAUFEY, 2010). Como resultado de esta disyunción, el sujeto no sabe lo que dice; no sabe si dice la verdad o si miente.

Retomando el eje de este apartado, es decir, el empleo de la expresión sujeto de la enunciación en la enseñanza lacaniana, advertimos que, al igual que lo que ocurre con la noción de enunciación, esta aparece con mayor frecuencia entre el Seminario 6 (2014) y el Seminario 17 (2006), para ser nuevamente mencionada en el Seminario 24 (s.f.f). El Seminario 14 (s.f.d) y el Seminario 15 (s.f.e), por otra parte, son aquellos donde se evidencia un mayor número de referencias sobre este sujeto: nueve y siete respectivamente. También es utilizada en "Observaciones sobre el informe de Daniel Lagache: "Psicoanálisis y estructura de la personalidad" (2008d), "Kant con Sade" (2008b), "Subversión del sujeto y dialéctica del deseo en el inconsciente freudiano" (2008g) y "La ciencia y la verdad" (2008c).

Es durante la clase del 3 de diciembre de 1958 la primera vez en la que -a partir de la noción del shifter perteneciente a la linguiística- se diferencia el yo (je) en tanto sujeto del enunciado y el yo (je) en tanto que sujeto de la enunciación $\left(2014\right.$, p.85) ${ }^{10}$. Para dar cuenta de esta distinción se recurre al ejemplo de la escala de Binet en la que se afirma: "Tengo tres hermanos, Pablo, Ernesto y yo". Este ejemplo permite vislumbrar que el niño se incluye en esta frase como un elemento más, se cuenta dentro del conjunto, debido a que no puede discriminar el yo del enunciado del yo de la enunciación. El sujeto se constituye, señala Lacan, justamente en el proceso de la diferenciación de ese yo.

Otro de los ejemplos a los que se apela para clarificar el contraste entre estos dos sujetos y que insiste en muchas de las citas encontradas es el uso del ne expletivo francés, un ne que no implica negación. La frase de la que se vale para echar luz sobre esta cuestión es Je crains qu'il ne vienne, que en español significa: "Temo que él venga". Este ne, siguiendo el planteo del psicoanalista francés, refleja la discordancia entre el enunciado y la enunciación, ya que se conjuga el temor de que él venga y el deseo de que no venga (2014, p.98).

\footnotetext{
${ }^{10}$ Algunos trabajos atribuyen erróneamente esta distinción a Benveniste.
} 
Ahora bien, para alcanzar una caracterización más acertada sobre el sujeto de la enunciación es necesario reflexionar respecto del modo en que este puede ser articulado con las tres vertientes de la enunciación que hemos presentado en el punto anterior y que entendemos que están implicadas en esta expresión.

Este sujeto podría ubicarse, en primer término, en el acto de la enunciación. En este sentido, el acto determina que el sujeto se divida entre su enunciación y su enunciado, lo que nos reenvía a la noción de sujeto dividido que hemos mencionado:

\begin{abstract}
Es lo que nos permite avanzar y afirmar que la distinción de la operación cuantificadora, cuando la ponemos en su función rectora, función de régimen de la operación lógica, se distingue de la lógica de Aristóteles a la que sustituye, en el lugar donde la ousia, la esencia, lo ontológico no es eliminado, en el lugar del sujeto gramatical, el sujeto que nos interesa en tanto sujeto dividido, a saber la pura y simple división como tal del sujeto en tanto que habla, del sujeto de la enunciación en tanto que distinto del sujeto del enunciado. (s.f.e, p. 140)
\end{abstract}

Al enunciar, el sujeto se escinde entre el yo (je) del enunciado -que apunta a la ilusión de unidad a la que nos referimos previamente- y el yo (je) de la enunciación. Vemos aquí que el sujeto no puede ser previo a ese acto, sino que se constituye en la enunciación misma, está sujetado a ella, y, por ello, al lenguaje: "escisión del sujeto que se opera por toda intervención del significante: concretamente del sujeto de la enunciación al sujeto del enunciado." (2008b, p. 732).

Dicho de otra manera, el sujeto nace de esta división, que -insistimos- surge de este acto, al proferir un enunciado.

En segunda instancia, el sujeto puede correlacionarse con la enunciación en tanto dimensión. Si existen dos planos en toda producción linguiística, el plano del enunciado y el plano de la enunciación, también pueden rastrearse allí el sujeto de ambas dimensiones: un sujeto relativo al nivel de la conciencia y otro anclado en el nivel del inconsciente. Este segundo sujeto es el que el psicoanalista describe como indeterminado (s.f.a), dado que es un sujeto que falta en el enunciado:

\footnotetext{
He podido mostrarles el año último lo que concierne en su función central a este término de indicación cuyo tipo es el shifter lo que está esencialmente indicado es siempre más o menos, el agujero del sujeto de la enunciación. (s.f.c, p. 21)
}

Este conjunto vacío en tanto representa al sujeto de la enunciación (s.f.d, p. 29)

¿Cómo podrían interpretarse estas citas? Por un lado, vinculadas con el primer sentido del término enunciación, se pueden correlacionar con la noción de sujeto dividido en el acto de su enunciación y, por el otro, con la idea de que el sujeto de la enunciación no está presente explícitamente en el enunciado, en tanto -podríamos agregar-pertenece al plano inconsciente. En esta dirección pueden leerse las palabras de Lacan en "Subversión del sujeto y dialéctica del deseo en el inconsciente freudiano" (2008g, p.761); artículo en el que asegura que el shifter que indica el sujeto del enunciado designa también al sujeto de la enunciación, pero que no lo significa. 
Esta falta es, asimismo, aquello que determina el desconocimiento de ese sujeto y su incapacidad de reflexividad:

\begin{abstract}
Esto está hecho para hacernos interrogar sobre lo que hay de eso en este punto radical, araico, que tenemos necesidad de suponer en el origen del inconsciente, es decir, eso por lo cual en tanto el sujeto habla, no puede sino avanzar siempre más adelante en la cadena, en el desarrollo de los enunciados, pero que dirigiéndose hacia los enunciados, por ese hecho mismo, en la enunciación elide algo que es hablando con propiedad lo que no puede saber, a saber, el nombre de lo que él es en tanto sujeto de la enunciación. (s.f.a, p.71)
\end{abstract}

Ahora bien, esta idea de dimensión o plano resulta bastante general y, por lo tanto, imprecisa y vaga, en particular para el trabajo analítico. Es el tercer sentido que hemos hallado en referencia a la enunciación el que nos permite, en verdad, afinar y ajustar la expresión sujeto de la enunciación. En efecto, entender la enunciación como cadena nos orienta en la comprensión y, fundamentalmente, en el encuentro con ese sujeto. El sujeto de la enunciación se localiza en ese otro enunciado que nace de la enunciación y que atraviesa el enunciado efectivamente dicho. Adquiere, en otras palabras, su "soporte" (1990b, p.265) en ese segundo enunciado, anclando su sentido en esta segunda cadena. En otras palabras, este sujeto que es consecuencia del acto de enunciar, que está ausente del enunciado, puede advenir en el punto en el que el enunciado y la enunciación se encuentran. De allí que el lenguaje no solo opere en la división del sujeto, sino también en su irrupción: "El lenguaje no es un código porque en su enunciado vehiculiza al sujeto presente en su enunciación." (s.f.b., p. 88).

Por este motivo, Lacan plantea que el sujeto de la enunciación está implicado o implícito en el enunciado:

\begin{abstract}
Les puse el acento sobre la diferencia entre el yo [je] del enunciado y el yo [je] de la enunciación. El primero está implicado en cualquier enunciado, en la medida en que, al igual que cualquier otro, es el sujeto de una acción enunciada, por ejemplo, lo cual no es por otra parte el único modo de enunciado. El otro está implicado en toda enunciación, pero tanto más cuando se anuncia como yo de la enunciación. (2014, p. 93)
\end{abstract}

Fomentamos este año uno de nuestro cuño, en el que creemos poder designar el lecho en que oscilan entre una cadena de la enunciación en cuanto que marca el lugar donde el sujeto está implícito en el puro discurso [...], y una cadena del enunciado en cuanto al sujeto está designado en ella por los shifters (2008d, p.632)

Las ideas de acto, del agujero, de la ausencia-presencia, relativas al sujeto de la enunciación, imprimen, entonces, su carácter volátil y fugitivo.

en el plano del sujeto de la enunciación, en la medida en que se, según las frases, según los modos, éste se pierde tanto como se vuelve a encontrar, y que, en una interjección, en un imperativo, en una invocación, y aun en un desfallecimiento, siempre es él quien la afirma a uno su enigma, y quien habla (2001, p.34)

De esta manera, a la vez que se asoma en algún punto de la cadena significante, de la cadena del enunciado, se desvanece nuevamente, dejando, por ello, su enigma, que como indicamos- es pura enunciación. 
Luego del recorrido por los trabajos de Émile Benveniste y Jacques Lacan, estamos en condiciones de abordar el problema del sujeto de la enunciación para la lingüística, o, al menos, dejar asentado cierto esbozo. Partamos, para ello, de la existencia de diversas categorías que suelen circular entre las distintas teorías sobre el lenguaje, tales como, emisor, hablante, sujeto hablante, sujeto de conciencia, locutor, enunciador y, la que aquí nos interroga, sujeto de la enunciación, entre otras. Estas categorías, muchas veces empleadas incorrectamente como sinónimos, poseen su propia especificidad y adquieren valor dentro de los diferentes marcos teóricos. No obstante, intentaremos realizar algunos señalamientos que contribuyan a revisar algunos puntos que consideramos medulares.

Recordemos el clásico trabajo de Oswald Durcot (2001) sobre la teoría polifónica de la enunciación. Este lingüista, quien alude al sujeto de la enunciación como el autor que tiene a cargo el enunciado, distingue entre locutores y enunciadores. El locutor es al que se le imputa la responsabilidad del enunciado, no necesariamente es el autor real. Discrimina el "locutor como tal" (L), responsable de la enunciación, y el "locutor como ser del mundo" $(\lambda)$, la persona "completa" donde se origina el enunciado. Por otra parte, los enunciadores son los seres que se expresan a través de la enunciación; es decir, la enunciación se presenta como si fuese la manifestación de su punto de vista. Es interesante marcar que este ordenamiento -que se contrapone con la perspectiva psicoanalítica por diversas cuestiones, pero fundamentalmente por la tesis de que, según el lingüista, es el locutor el responsable de la enunciación- plasma, sin embargo, la existencia de una división subjetiva. En efecto, polemizando con la posición que sostiene el carácter unitario del sujeto, Ducrot perfila un sujeto dividido en el acto de su enunciación.

La concurrencia de diversos enunciadores en un enunciado, entendida como la presencia simultánea de distintas voces en el interior de lo dicho, resulta evidente en diferentes fenómenos lingüísticos ${ }^{11} \mathrm{y}$, en este sentido, la búsqueda de las huellas de esta emergencia es una tarea de la que se ha ocupado y se ocupa la lingüística y el análisis del discurso. No nos adentraremos aquí -no nos resulta pertinente-en los numerosos estudios que buscan dar cuenta del modo en que se configura la voz del enunciador al que se le atribuye determinada enunciación, ni a la dificultades que esta categoría promueve ${ }^{12}$. Nos interesa, por el contrario, establecer su articulación con el sujeto de la enunciación considerando fundamentalmente su denominación.

El término enunciador, ligado a la instancia construida por el enunciado en su relación con la situación de enunciación, presenta un carácter activo si lo pensamos como "aquel que enuncia". Por tal razón, localizar esa voz implica rastrear en el "texto" este "ser textual" que, paradójicamente, enuncia y es enunciado, habla y es hablado. En contrapartida, la expresión sujeto de la enunciación, que Filinich (2005) define como una instancia que articula enunciador y enunciatario, va más allá de la figura del enunciador, ya que pone el acento, a nuestro entender, en su condición de "ser sujetado por la

\footnotetext{
${ }^{11}$ No podemos dejar de destacar los trabajos de Jacqueline Authier-Revuz en este punto (1984).

12 Véase, por ejemplo, la entrada enunciador del diccionario elaborado por Charaudeau y Maingueneau (2005)
} 
enunciación", es decir, en su condición de ser un efecto del acto enunciativo, una consecuencia del lenguaje y su puesta en funcionamiento.

Ahora bien, si adoptamos una visión ampliada de la noción de enunciación (KERBRAT-ORECCHIONI, 1997), que incluya su aspecto discursivo, y si la articulamos con la perspectiva lacaniana que nos reenvía al discurso del Otro y a la enunciación entendida como un Otro enunciado, podríamos pensar, entonces, una enunciación atravesada por otros enunciados, otros discursos: sociales, históricos, económicos, antropológicos, entre muchos otros. Siguiendo este planteo, podríamos afirmar que el sujeto de la enunciación no es solamente un efecto del lenguaje, sino también un efecto de estos otros enunciados que dejan trazos en el devenir discursivo ${ }^{13}$. Ir al encuentro de este sujeto de la enunciación significa rastrear sus entrecruzamientos con esas otras voces, esos otros enunciados, ubicar sus puntos de discordancia, en fin, los lugares, -tomando términos de Lacan- en los que el enunciado y la enunciación se confunden y se embrollan.

Esta posición en torno al sujeto de la enunciación contiene, en principio, tres implícitos. En primer lugar, supone un sujeto barrado, dividido por el acto significante, que no necesariamente está advertido de esas otras voces que subyacen en su decir. En segundo lugar, no se pregunta por la existencia o no de un sujeto previo al discurso, dado que encierra la idea de un sujeto que emerge en el enunciado, que puede localizarse allí y que, por lo tanto, puede analizarse ${ }^{14}$. Por último, refiere a un sujeto que, al estar ligado al acto enunciativo, dura el instante de ese acto. Y como plantea Lacan, se pierde en la medida en que se encuentra. En ese sentido, carece de la condición de inmutabilidad. Es, en esencia, heterogéneo.

\section{PALABRAS FINALES}

En este artículo, hemos buscado poner a dialogar la lingüística y el psicoanálisis a partir de pensar el sujeto de la enunciación. Nuestro objetivo fue ubicar el aporte de los desarrollos teóricos lacanianos a los estudios sobre la enunciación, con el fin de reflexionar en relación con la ambigüedad que muchas veces subyace a esta expresión, que oscila entre un sujeto pleno y un sujeto clivado.

El análisis de la noción de sujeto que circula en Benveniste - del que se desprende que este puede ser entendido en función del lenguaje, el discurso, el acto y el otro- nos habilita a articularlo con el sujeto lacaniano. En efecto, a pesar de que el lingüista no elabore una teoría del sujeto ni emplee la expresión sujeto de la enunciación, el sujeto al que alude es un sujeto que no puede concebirse sino es a partir de su vinculación con el significante: es efecto del lenguaje, se halla en el discurso y está dividido por él, y nace en el acto enunciativo. Aquello que hace tope y que lo distancia del planteo psicoanalítico es, en verdad, el plano de la intersubjetividad. La simetría que postula entre el sujeto y el otro se aparta del pensamiento lacaniano.

\footnotetext{
${ }^{13}$ Esto claramente se relaciona con la noción de interdiscurso.

${ }^{14}$ En este punto disentimos con lo que plantean Flores y Teixeira (2013) sobre la complejidad de analizar el sujeto de la enunciación en tanto involucra aspectos exteriores a la lingüística. De la manera en que concebimos el sujeto en este artículo su análisis se desprende del estudio de la enunciación.
} 
Por otra parte, las tres vertientes que advertimos en torno a la enunciación -el acto, la dimensión y la cadena- nos permiten especificar el modo el que el psicoanalista hace uso del sujeto de la enunciación y observar la novedad de la propuesta lacaniana. Este sujeto es pensado, así, desde tres lugares: como un sujeto dividido por el acto enunciativo y como efecto de esta división; como un sujeto indeterminado, que no sabe; y como un sujeto soportado e implicado en la cadena significante.

Este sujeto, entonces, "sujetado" por el lenguaje, que en vez de hablar es hablado por ese Otro del discurso puede ser recuperado por los estudios enunciativos inscribiéndolo en un enfoque que supere el vínculo del enunciado con su aquí y ahora, a partir de considerar la enunciación como otros enunciados "no sabidos" que se entrecruzan en el decir de un sujeto y que, por ello, complejizan la figura del enunciador.

\section{REFERENCIAS}

ARRIVÉ, M. Lingüística y psicoanálisis. México D.F.: Siglo Veintiuno Editores, 2001.

AUTHIER-REVUZ, J. Hétérogénéité(s) énonciative(s). Langages, n. 73, p .98-111, mars 1984.

BENVENISTE, É. Problemas de lingüística general. Tomo I. México: Siglo XXI, 2004a. Problemas de lingüística general. Tomo II. México: Siglo XXI, 2004b.

CHARAUDEAU, P.; MAINGUENEAU, D. Diccionario de análisis del discurso. Buenos Aires: Amorrortu, 2005.

DUCROT, O. El decir y lo dicho. Buenos Aires: Edicial, 2001.

FILINICH, M. I. Enunciación. Buenos Aires: Eudeba, 2005.

FLORES, V. do N.; TEIXEIRA, M. Introdução à Linguística da Enunciação. São Paulo: Contexto, 2013. FLORES, V. Linguística e psicanálise: princípios de uma semântica da enunciação. Porto Alegre: EDIPUCRS, 1999.

JAKOBSON, R. Les embrayeurs, les cátegories verbales et le verbe russe. En: Essais de linguistique générale. Paris: Minuit, 1963, p. 176-196.

KERBRAT-ORECCHIONI, C. La enunciación. De la subjetividad en el lenguaje. Buenos Aires: Edicial, 1997.

KRISTEVA, J. Du sujet en linguistique. Langages, n. 24, p. 107-126, 1971.

Prefacio. En: BENVENISTE, E. Últimas lecciones. Collège de France. 1968-1969. Buenos Aires: Siglo XXI, 2014, p.17-51.

LACAN, J. Función y campo de la palabra y del lenguaje en psicoanálisis. En: Escritos, t.1. Buenos Aires: Siglo XXI, 2008a, p.231-309.

LACAN, J. Kant con Sade. En: Escritos, t.2. Buenos Aires: Siglo XXI, 2008b, p.727-751.

La ciencia y la verdad. En: Escritos, t.2. Buenos Aires: Siglo XXI, 2008c, p.813-834.

Observaciones sobre el informe de Daniel Lagache: "Psicoanálisis y estructura de personalidad".

En: Escritos, t.2. Buenos Aires: Siglo XXI, 2008d, p. 617-651. Posición del inconsciente. En: Escritos, t.2. Buenos Aires: Siglo XXI, 2008e, p.789 -808.

. Seminario 3. Las Psicosis. Buenos Aires: Paidós, 1990a.

Seminario 6. El deseo y su interpretación. Buenos Aires: Paidós, 2014.

Seminario 7. La ética del Psicoanálisis. Buenos Aires: Paidós, 1990b.

Seminario 9. La identificación. Inédito. Buenos Aires: Escuela Freudiana de Buenos Aires, s.f.a.

2001.

Seminario 11. Los cuatro conceptos fundamentales del psicoanálisis. Buenos Aires: Paidós,

Seminario 12. Problemas cruciales para el psicoanálisis. Inédito. Buenos Aires: Escuela

Freudiana de Buenos Aires, s.f.b. Aires, s.f.c.

Seminario 13. El objeto del psicoanálisis. Inédito. Buenos Aires: Escuela Freudiana de Buenos 
Seminario 14. Lógica del fantasma. Inédito. Buenos Aires: Escuela Freudiana de Buenos Aires, s.f.d.

Seminario 15. El acto psicoanalítico. Inédito. Buenos Aires: Escuela Freudiana de Buenos Aires, s.f.e.

Seminario 16. De un otro al otro. Buenos Aires: Paidós, 2008f.

Seminario 17. El reveso del psicoanálisis. Buenos Aires: Paidós, 2006.

Seminario 24. L'insu que sait de l'une-bévue s'aile à mourre. Inédito. Buenos Aires: Escuela Freudiana de Buenos Aires, s.d.f.

Subversión del sujeto y dialéctica del deseo en el inconsciente freudiano. En: Escritos, t.2.

Buenos Aires: Siglo XXI, 2008g, p.755-787.

LE GAUFEY, G. El sujeto según Lacan. Buenos Aires: El cuenco de plata, 2010.

MILNER, J.-C. El amor por la lengua. México D.F.: editorial Nueva Imagen, 1980.

El periplo estructural. Figuras y paradigma. Buenos Aires: Amorrortu, 2003.

NORMAND, C. Le sujet dans la langue. Langages, n. 77, p. 7-19, 1985.

NORMAND, C. Lectures de Benveniste : quelques variantes sur un itinéraire balisé. Linxs, n. 9, p. 1-12, julio 1997. 206, 1986

Les termes de l'énonciation de Benveniste. Histoire Épistémologie Langage, t. 8, n. 2, p. 191-

OGILVIE, B. Lacan. La formación del concepto de sujeto (1932-1949). Buenos Aires: ediciones Nueva Visión, 2000.

TODOROV, T. Posfacio. Émile Benveniste, el destino de un estudioso. En: BENVENISTE, E. Últimas lecciones. Collège de France. 1968-1969. Buenos Aires: Siglo XXI, 2014, p. 203-220.

Recebido em: 10/02/17. Aprovado em: 08/07/17.

Title: The subject of the enunciation: dialogues between linguistics and psychoanalysis

Author: Karina Savio

Abstract: This article intends to look over the expression subject of the enunciation in the work by Jacques Lacan. As in the studies that deal with the problem of the enunciation there is no consensus around which subject is referred to, we recover the psychoanalytic origin of this term with the aim of introducing some reflections. This way has led us, firstly, to trace the notion of subject in Benveniste, and then enunciation in the Lacanian texts. The analysis has determined the coexistence of three aspects around the enunciation that allow us to consider different dimensions with respect to how the subject of the enunciation is conceived.

Keywords: Psychoanalysis. Lacan. Subject. Enunciation.

Título: O sujeito da enunciação: diálogos entre a linguística e a psicanálise

Autora: Karina Savio

Resumo: Neste artigo propõe-se realizar um estudo da expressão sujeito da enunciação na obra de Jacques Lacan. Tendo em vista que nos estudos em que é abordada a problemática da enunciação não existe consenso com respeito a que sujeito se faz referência, recuperamos a origem psicanalítica desse termo, com o objetivo de propor algumas reflexões. Esse caminho nos conduziu, como primeiro passo, a rastrear a noção de sujeito em Benveniste e, em seguida, de enunciação nos textos lacanianos. A análise determinou a coexistência de três vertentes com referência à enunciação, que permitem introduzir diferentes dimensões com respeito a como se concebe o sujeito da enunciação.

Palavras-chave: Psicanálise. Lacan. Sujeito. Enunciação.

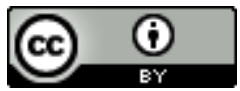

Este texto está licenciado com uma Licença Creative Commons Atribuição 4.0 Internacional. 